\title{
Nonlinear Opto-mechanics Using Radiation Pressure in High-Q Microcavities
}

\author{
Kerry Vahala†, Tobias Kippenberg*, Tal Carmon $\dagger$, Mani Hossein Zadeh $\uparrow$ \\ $\uparrow$ California Institute of Technology, Pasadena, CA 91125 \\ *Max Planck Institut für Quantenoptik, Garching 85748, Germany \\ vahala@caltech.edu
}

\begin{abstract}
Recent experiments that use radiation pressure in microcavities either to create micro-wave-rate mechanical oscillations, or to cool a mechanical degree of freedom to cryogenic temperatures are overviewed. The implication of these results for new science is discussed.
\end{abstract}

Recent years have witnessed a series of developments at the intersection of two, previously distinct subjects. Optical microcavities and micro (nano) mechanical resonators, each a subject in its own right with a rich scientific and technological history [1,2], have, in a sense, become entangled experimentally. The results have implications in a wide range of subjects including improved gravity wave detection [3] and new tests of quantum theory [4]. They also suggest the beginning of an exciting period of experimental science.

Central to these new results have been two device geometries that enable structural coexistence of micro-mechanical and optical resonators. In one geometry, a micro-cantilever mechanical resonator also functions as a mirror in a high-finesse optical cavity. In a second, opto-mechanical coexistence takes the form of a micron-scale silica toroid that exhibits both high-Q radio-frequency mechanical resonances and optical resonances with Q's as high as 500 million [5]. In both cases, the pressure of photons circulating within the optical resonator couples the mechanical and optical degrees of freedom. Although the static effect of this coupling was measured nearly two decades ago [6], there exist dynamical phenomena that have only recently been observed and that enable new, opto-mechanical physics. The first of these is the onset of regenerative mechanical oscillation caused by radiation pressure. This so-called parametric instability [3] was first observed in silica microtoroids [7] and the resulting mechanical oscillations have now been observed from radio-frequency to micro-wave rates. This oscillation phenomenon is a manifestation of the more general principle of dynamic back action [3], and has a counterpart in which laser cooling of the mechanical mode is possible [3,7]. Recent demonstrations of cooling to Kelvin temperatures by this method [8] and by a related method of feedback cooling [9] will be briefly reviewed.

In addition to providing a powerful set of tools for nano-mechanics [2], these results establish a new direction of basic studies in opto-mechanics. Beyond the new science, cooling and regenerative 
oscillation on a silicon chip (as in the case of a microtoroid) can one-day enable new applications in microchip technologies.

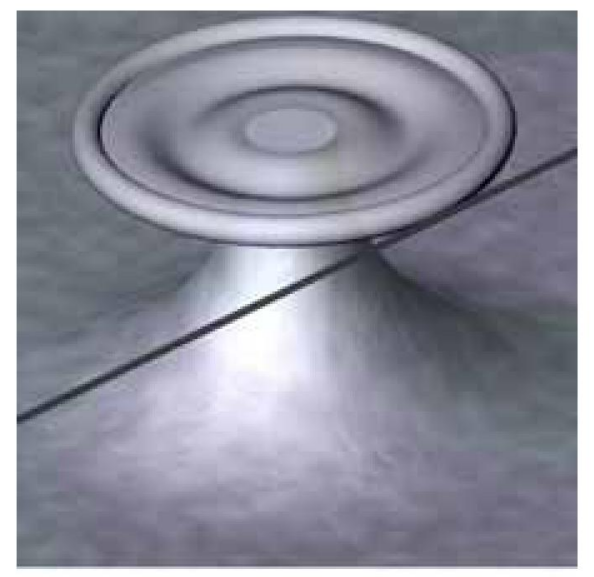

Figure: Rendering of a toroidal optical resonator exhibiting mechanical oscillation driven by radiation pressure. Black line is fiber taper waveguide used to couple the CW pump wave.

\section{References}

[1] K. Vahala, "Optical Microcavities," Nature, vol. 424, No. 6950, August 2003.

[2] K. Schwab and M L Roukes, "Putting Mechanics into Quantum Mechanics," Physics Today, July 2005.

[3] V. B. Braginsky, S. P. Vyatchanin, Phys. Lett. A, 293, 228 (2002).

[4] S. Mancini et. al., Phys. Rev Lett., 88, no. 12, 120401-1 (2002); Marshall, W. et. al. Phys. Rev. Lett., 91, 130401 (2003).

[5] D. K. Armani, et. al. Nature, 421, pp. 925-929, 27 February (2003).

[6] Dorsel, A, McCullen, J., Meystre, P., Vignes, E. \& Walther, H., Phys. Rev. Lett. 51, 1550-1553 (1983).

[7] T. J. Kippenberg, et. al. Phys. Rev. Lett. 95, 033901, 2005. ; T. Carmon, et. al., Phys. Rev. Lett., 94, 223902, June 2005.; H. Rokhsari, et. al. Optics Express, 13, No. 14, July 2005.

[8] A. Schliesser, et. al. Phys. Rev. Lett., 97, 243905, Dec 15, (2006); S. Gigan, H.R. et. al., Nature (London) 444, 67 (2006); O. Arcizet, et. al., Nature (London) 444, 71 (2006).

[9] Kleckner, D.\& Bouwmeester, D., Nature 432, 75-78 (2006). 